\title{
Fractional Calculus-Based Modeling of Electromagnetic Field Propagation in Arbitrary Biological Tissue
}

\author{
Pietro Bia, ${ }^{1}$ Luciano Mescia, ${ }^{1}$ and Diego Caratelli ${ }^{2,3}$ \\ ${ }^{1}$ Dipartimento di Ingegneria Elettrica e dell'Informazione, Politecnico di Bari, Via E. Orabona 4, 70125 Bari, Italy \\ ${ }^{2}$ The Antenna Company Nederland B.V., High Tech Campus, 5656 AE Eindhoven, Netherlands \\ ${ }^{3}$ The Institute of Cybernetics, Tomsk Polytechnic University, 84/3 Sovetskaya Street, Tomsk 634050, Russia \\ Correspondence should be addressed to Pietro Bia; pietro.bia@poliba.it
}

Received 2 March 2016; Revised 25 September 2016; Accepted 3 November 2016

Academic Editor: Ivan D. Rukhlenko

Copyright (C) 2016 Pietro Bia et al. This is an open access article distributed under the Creative Commons Attribution License, which permits unrestricted use, distribution, and reproduction in any medium, provided the original work is properly cited.

\begin{abstract}
The interaction of electromagnetic fields and biological tissues has become a topic of increasing interest for new research activities in bioelectrics, a new interdisciplinary field combining knowledge of electromagnetic theory, modeling, and simulations, physics, material science, cell biology, and medicine. In particular, the feasibility of pulsed electromagnetic fields in RF and mm-wave frequency range has been investigated with the objective to discover new noninvasive techniques in healthcare. The aim of this contribution is to illustrate a novel Finite-Difference Time-Domain (FDTD) scheme for simulating electromagnetic pulse propagation in arbitrary dispersive biological media. The proposed method is based on the fractional calculus theory and a general series expansion of the permittivity function. The spatial dispersion effects are taken into account, too. The resulting formulation is explicit, it has a second-order accuracy, and the need for additional storage variables is minimal. The comparison between simulation results and those evaluated by using an analytical method based on the Fourier transformation demonstrates the accuracy and effectiveness of the developed FDTD model. Five numerical examples showing the plane wave propagation in a variety of dispersive media are examined.
\end{abstract}

\section{Introduction}

Recently, multifrequency and ultrawideband microwave technologies have receiving increasing attention due to their promising applications in biological and medical domains [13]. Several studies concern microwave dielectric spectroscopy and imaging since their represent potential solutions for early-stage cancer detection and treatment as well as for cancer investigations and diagnostic [4, 5]. Many research activities are focused on the use of electromagnetic waves for discriminating malignant tissues from healthy ones. Moreover, recent applications employing electromagnetic technology include medical implant communication service, wireless medical telemetry service, and medical body area network [6-8]. Electromagnetic wave technologies have also been introduced as a rapid method of delivering high temperatures to destroy the cancer cells during thermal ablation [9]. Finally, continuous and pulsed electric field are successfully used in a variety of therapeutic and diagnostic applications such as hyperthermia, electroporation, and treatment of specific diseases [10].

All these technologies involve the interaction of electromagnetic fields with living tissues. As a result, detailed theoretical modeling and computational techniques are essential to gain insight into the several phenomena occurring within the biological materials subject to an imposed electromagnetic field. For example, the right knowledge of the electromagnetic field distribution inside biological tissues is necessary to demonstrate the effects of electromagnetic radiation absorption. Furthermore, numerical simulations under various conditions can be used to identify the fundamental parameters involved in noninvasive diagnosis and medical sensors as well as to provide guidance for computational dosimetry and for the development of specific therapeutic approaches.

In recent decades, fractional calculus has been a fruitful field of research in science and engineering. It has found use in studies of viscoelastic materials and anomalous diffusion 
processes, as well as in many fields of science and engineering including signal processing, mathematical biology, electrochemistry, electrical networks, electromagnetic theory, and probability [11-15]. As well known, the concept of fractional exponents is an outgrowth of exponents with integer value. In the same way, nonintegral order of integration is a generalization of the mathematical operations of differentiation and integration to arbitrary, general, noninteger order. Although it better models the higher complexity by nature, it is still fairly easy to physically represent its meaning. However, just as fractional exponents may find their way into innumerable equations and applications, it will become apparent that integrations of fractional order can find practical use in many modern problems. Taking in consideration that the study of fractional calculus opens the mind to entirely new branches of thought, we applied such concept in a specific electromagnetic problem in order to demonstrate that the fractional derivatives operator can be an interesting and useful tools in electromagnetic theory.

Biological tissues are heterogeneous mixtures of various materials and quantities such as water, ions, membranes, and macromolecules with a broad variety of profiles. Therefore, their interaction with electromagnetic radiation takes place in different relaxation processes including (i) reorientation of water and protein-bound water molecules, (ii) interfacial polarization arising by the presence of two or more regions with different electrical properties, (iii) ionic diffusion, (iv) tumbling motion of the protein molecules, and (v) relaxations due to the nonspherical shape. These phenomena cause a frequency dispersion pattern of permittivity and conductivity which is characterized by a series of steps as the frequency increases [16, 17].

The Debye model has been widely used to describe the dielectric response arising by the dipolar relaxation [18, 19]. However, this simple exponential law cannot describe a wide class of relaxation processes occurring in biological materials since the intrinsic structural disorder and the ion-ion interaction induce anomalous dynamics based on nonexponential temporal decay. As a consequence, a number of empirical dispersion functions, such as Cole-Cole, ColeDavidson, Havriliak-Negami, and Raicu spectral models, have been developed to accurately fit the fractional powerlaw decays of the dielectric response [17].

Finite-Difference Time-Domain (FDTD) method has been widely used in electromagnetic simulation due to its straightforward implementation and ability to model a broad range of initial and boundary values problems. Taking into account that the dielectric response includes fractional powers of $j \omega$, the design of the FDTD algorithm requires special treatments aimed at embedding the approximation of fractional derivatives into the simulator [20-23].

Besides the requirements linked to the frequency behavior of dispersive materials, a complete numerical code should be able to correctly incorporate, inside its kernel, the spatial dependence of the material parameters. This is a fundamental skill to meet especially when biological tissues have to be employed in the simulation. In fact, from the macroscopic point of view, a biological tissue can be treated as a random mixture of different medium compounds having a spatialdependent concentration.

Considering these premises and the requirement to model a more general permittivity function, in this paper we illustrate a more accurate study of the time-domain electromagnetic field propagation in arbitrary dispersive media. The study is fully motivated to seek for an extended model flexibility enabling a better parametrization of the arbitrary dispersive media properties as well as a better fitting, over broad frequency ranges, of the experimental dielectric response. In particular, using a general fractional polynomial series approximation and the fractional calculus theory both the spatial and frequency dispersion characteristics of the dielectric response have been incorporated into the developed FDTD scheme. Dedicated uniaxial perfectly matched layer boundary conditions have been also derived and implemented in combination with the basic time-marching scheme. In particular, the iterative E-field and $\mathbf{H}$-field updating while applying boundary conditions results in a marching-in-time procedures that simulate the continuous actual electromagnetic waves in a finite spatial region by sampled-data numerical analogs propagating in a computer data space [24]. Moreover, ohmic losses and total field/scattered field approach are taken into account. The accuracy of the proposed FDTD scheme has been tested by considering several test cases involving different spatial and time-dependent permittivity profiles.

\section{Formulation of the Proposed FDTD Method}

FDTD method is a powerful and efficient numerical technique used to solve both simple and complicated electromagnetic problems. This method has attracted much attention due to its simplicity, accuracy, robustness, low computational footprint, and its capability in treating in a straightforward and effective way many types of dispersive media. FDTD technique is based on the direct numerical integration of Maxwell's two curl equations [25]. In dielectric materials, such equations are as follows:

$$
\begin{aligned}
& \nabla \times \mathbf{H}=\frac{\partial \mathbf{D}}{\partial t}+\sigma \mathbf{E}+\mathbf{J}_{0} \\
& \nabla \times \mathbf{E}=-\mu_{0} \frac{\partial \mathbf{H}}{\partial t}
\end{aligned}
$$

where $\mathbf{D}$ is the electric flux density, $\mathbf{E}$ is the electric field, $\mathbf{H}$ is the magnetic field, $\mu_{0}$ is the magnetic permeability of free space, $\mathbf{J}_{0}$ is the source current density, and $\sigma$ is the static conductivity due to the ionic or electronic charge transport and modeling the ohmic losses. To calculate the spatial and temporal distributions of the electromagnetic fields in a medium, the constitutive relation between the fields $\mathbf{D}$ and $\mathbf{E}$ has to be combined with the equations (1). In frequency domain, the relative electric permittivity of a linear and nonmagnetic media can be expressed as $[25,26]$

$$
\varepsilon_{r}(\omega)=\frac{\varepsilon(\omega)}{\varepsilon_{0}}=\varepsilon_{\infty}+\chi(\omega),
$$


where $\varepsilon_{0}$ is the permittivity of free space, $\varepsilon_{\infty}$ is the relative permittivity at high frequency limit, and $\chi$ is the electric susceptibility.

FDTD methods have been developed for incorporating Debye, Cole-Cole, Cole-Davidson, and Havriliak-Negami electric susceptibility models [27-30]. The common approach is to approximate the relative complex permittivity by means of rational or polynomial functions resulting in auxiliary differential equation FDTD models. When implemented to predict the electromagnetic field propagation inside quite simple dispersive media, such numerical models provide reliable results. On the other hand, their effectiveness falls when more complex and multirelaxed dispersive media are employed since the approximation does not accurately fit the frequency behavior of the electric permittivity. In order to overcome this drawback, we have successfully implemented in the FDTD formulation a more general electric susceptibility function having the following fractional polynomial dispersion response [31]:

$$
\chi(\omega)=\frac{\Delta \varepsilon}{1+\sum_{p=1}^{N} A_{p}\left(j \omega \tau_{p}\right)^{\alpha_{p}}},
$$

where $\Delta \varepsilon=\varepsilon_{s}-\varepsilon_{\infty}$ is the relaxation strength with $\varepsilon_{s}$ being the relative permittivity at low frequency limit, $\alpha_{p} \geq$ $0, \tau_{p}$ is the relaxation time, and $A_{p}$ is a real coefficients related to the material parameters. The condition $A_{p} \geq 0$ is recommended to meet the requirement on the numerical stability of the developed time-marching scheme. Moreover, implementing an optimization algorithm based on swarm intelligence the parameters $A_{p}$ and $\alpha_{p}$ can be recovered to suitably fit a generic frequency domain permittivity function [32]. In particular, if $N_{\max }$ is the maximum expansion order in (3) and $\delta$ is a given small positive threshold to be used for controlling the accuracy of the approximation, a dedicated numerical procedure based on the enhanced weighted quantum particle swarm optimization (EWQPSO) was used to solve the minimization problem

$$
f_{\mathrm{err}}=\sqrt{\frac{\int_{\omega_{\min }}^{\omega_{\max }}|\bar{\chi}(\omega)-\chi(\omega)|^{2} \mathrm{~d} \omega}{\int_{\omega_{\min }}^{\omega_{\max }}|\chi(\omega)|^{2} \mathrm{~d} \omega}} \leq \delta
$$

where $\bar{\chi}(\omega)$ is the dispersion response to approximate. This technique has proved to feature superior effectiveness in terms of convergence rate and accuracy in comparison with alternative evolutionary stochastic search methods such as genetic algorithm [31, 32].

In linear dispersive materials, the frequency domain equation relating the electric and polarization fields is

$$
\mathbf{P}(\omega)=\varepsilon_{0} \chi(\omega) \mathbf{E}(\omega)
$$

or by substituting (3),

$$
\mathbf{P}(\omega)+\sum_{p=1}^{N} A_{p} \tau_{0}^{\alpha_{p}}(\mathrm{j} \omega)^{v_{p}}(\mathrm{j} \omega)^{\alpha_{p}-v_{p}} \mathbf{P}(\omega)=\varepsilon_{0} \Delta \varepsilon \mathbf{E}(\omega),
$$

where $v_{p}=\left\lceil\alpha_{p}\right\rceil$.
Taking the inverse Fourier transform of (6),

$$
\mathbf{P}(t)+\sum_{p=1}^{N} \frac{\partial^{v_{p}}}{\partial t^{v_{p}}} \int_{0}^{t} \zeta_{p}\left(t^{\prime}\right) \mathbf{P}\left(t-t^{\prime}\right) \mathrm{d} t^{\prime}=\varepsilon_{0} \Delta \varepsilon \mathbf{E}(t),
$$

where

$$
\zeta_{p}(t)=A_{p} \tau_{0}^{\alpha_{p}} \int_{-\infty}^{\infty}(\mathrm{j} \omega)^{\alpha_{p}-v_{p}} \mathrm{e}^{\mathrm{j} \omega t} \mathrm{~d} \omega
$$

Moreover, considering the gamma function, the following equation could be achieved (see Appendix A):

$$
\zeta_{p}(t)=\frac{A_{p} \tau_{0}^{\alpha_{p}}}{t^{\xi_{p}} \Gamma\left(1-\xi_{p}\right)}
$$

where $0<\xi_{p}=1+\alpha_{p}-v_{p}<1$. Differentiating both sides of (7) with respect to time and using Leibniz's rule for differentiation under the integral sign the following equation can be obtained:

$$
\mathbf{J}(t)+\sum_{p=1}^{N} \underbrace{\int_{0}^{t} \zeta_{p}\left(t^{\prime}\right) \frac{\partial^{v_{p}} \mathbf{J}}{\partial t^{v_{p}}} \mathrm{~d} t^{\prime}}_{\text {fractional derivatives }}=\varepsilon_{0} \Delta \varepsilon \frac{\partial \mathbf{E}(t)}{\partial t}
$$

where

$$
\mathbf{J}=\frac{\partial \mathbf{P}}{\partial t}
$$

is the polarization current. By using the constitutive relation

$$
\mathbf{D}(t)=\varepsilon_{0} \varepsilon_{\infty} \mathbf{E}(t)+\mathbf{P}(t)
$$

Ampere's law in time domain can be written as

$$
\begin{aligned}
\nabla \times \mathbf{H} & =\varepsilon_{0} \varepsilon_{\infty} \frac{\partial \mathbf{E}}{\partial t}+\mathbf{J}+\sigma \mathbf{E}+\mathbf{J}_{0} \\
& =\frac{\varepsilon_{\infty}}{\Delta \varepsilon}\left(\varepsilon_{0} \Delta \epsilon \frac{\partial \mathbf{E}}{\partial t}\right)+\mathbf{J}+\sigma \mathbf{E}+\mathbf{J}_{0}
\end{aligned}
$$

Applying a second-order accurate finite-difference scheme, one readily obtains at the time instant $t=m \Delta t$

$$
\left.(\boldsymbol{\nabla} \times \mathbf{H})\right|^{m}=\left.\frac{\varepsilon_{\boldsymbol{\infty}}}{\Delta \varepsilon}\left(\varepsilon_{0} \Delta \epsilon \frac{\partial \mathbf{E}}{\partial t}\right)\right|^{m}+\left.\mathbf{J}\right|^{m}+\left.\sigma \mathbf{E}\right|^{m}+\left.\mathbf{J}_{0}\right|^{m},
$$

where the vectors terms appearing on the right-hand side of (14) are evaluated using the semi-implicit approximation:

$$
\left.\mathbf{J}\right|^{m}=\frac{\left.\mathbf{J}\right|^{m-1 / 2}+\left.\mathbf{J}\right|^{m+1 / 2}}{2}
$$




$$
\begin{aligned}
\left.\mathbf{E}\right|^{m} & =\frac{\left.\mathbf{E}\right|^{m-1 / 2}+\left.\mathbf{E}\right|^{m+1 / 2}}{2}, \\
\left.\frac{\partial \mathbf{E}}{\partial t}\right|^{m} & =\frac{\left.\mathbf{E}\right|^{m+1 / 2}-\left.\mathbf{E}\right|^{m-1 / 2}}{\Delta t} .
\end{aligned}
$$

Evaluating (10) at $t=m \Delta t$ the following equation can be obtained (see Appendix B):

$$
\begin{aligned}
& \left.\mathbf{E}\right|^{m+1 / 2}=\left.\mathbf{E}\right|^{m-1 / 2}+\left.\frac{\Delta t}{\varepsilon_{0} \Delta \varepsilon}\left(R+\frac{1}{2}\right) \mathbf{J}\right|^{m+1 / 2} \\
& +\left.\frac{\Delta t}{2 \varepsilon_{0} \Delta \varepsilon} \mathbf{J}\right|^{m-1 / 2} \\
& +\frac{\Delta t}{\varepsilon_{0} \Delta \varepsilon}\left[\left.\sum_{p=1}^{N} \sum_{n=1}^{v_{p}} \frac{\eta_{p, 0}}{(\Delta t)^{v_{p}}}(-1)^{n}\left(\begin{array}{c}
v_{p} \\
n
\end{array}\right) \mathbf{J}\right|^{m-n+1 / 2}+Q\right] .
\end{aligned}
$$

Finally, by carrying out a second-order accurate finitedifference approximation of Faraday's law in the time domain, the following update equation for the magnetic field is obtained:

$$
\left.\mathbf{H}\right|^{m+1}=\left.\mathbf{H}\right|^{m}-\left.\frac{\Delta t}{\mu_{0}}(\nabla \times \mathbf{E})\right|^{m+1 / 2}
$$

In (14) and (17) the curl of magnetic and electric fields are approximated by the standard Yee algorithm. In our computations, a uniform space-time grid is employed. Moreover, a flowchart summarizing the main steps of the proposed FDTD algorithm is shown in Figure 1, where $m$ and $n$ represent the time and space indexes, respectively.

The arbitrary biological tissue can be generally visualized as a heterogeneous material. As a result, its dielectric properties are governed by a spatial average which can be expressed through an appropriate spatial dependence of the material parameters. To this aim, both Maxwell-Garnet and Bruggeman models have been taken into account [33]. In particular, in (2)-(3) the electric permittivity at both low and high frequency limits is expressed as

$$
\varepsilon_{a}(x)=\varepsilon_{a, m}\left[1+\frac{f(x)}{3} \frac{\sum_{i=i}^{3}\left(\left(\varepsilon_{a, 1}-\varepsilon_{a, m}\right) /\left(\varepsilon_{a, m}+N_{i}\left(\varepsilon_{a, 1}-\varepsilon_{a, m}\right)\right)\right)}{1-(f(x) / 3) \sum_{i=1}^{3}\left(N_{i}\left(\varepsilon_{a, 1}-\varepsilon_{a, m}\right) /\left(\varepsilon_{a, m}+N_{i}\left(\varepsilon_{a, 1}-\varepsilon_{a, m}\right)\right)\right)}\right], \quad a=s, \infty
$$

for Maxwell-Garnet model, and

$$
\begin{array}{r}
\varepsilon_{a}(x)=\varepsilon_{a, m}+\frac{f(x)}{3} \sum_{i=1}^{3} \frac{\varepsilon_{a}(x)}{\varepsilon_{a}(x)+N_{i}\left[\varepsilon_{a, 1}-\varepsilon_{a}(x)\right]}, \\
a=s, \infty,
\end{array}
$$

for Bruggeman model. In (18)-(19), $\varepsilon_{1}$ is the permittivity of inclusions having ellipsoidal shape, $\varepsilon_{m}$ is the permittivity of environment material, $0 \leq N_{i} \leq 1\left(N_{1}+N_{2}+N_{3}=1\right)$ is the depolarization factor, and $f(x)$ is the space-dependent filling factor function.

\section{Numerical Results}

To test the developed numerical code, the slab model illustrated in Figure 2 is implemented. It consists of a half space filled up with dispersive and nonhomogeneous biological material. The system is irradiated by a plane wave propagating along the positive $x$-direction, with electric field linearly polarized along the $y$-axis and located at a given point $x=x_{s}$. In particular, the time-domain signal source is

$$
\begin{aligned}
\mathbf{J}_{0}(x, t)= & \exp \left\{-\frac{f_{e}^{2}}{2}\left(t-\frac{2}{f_{e}}\right)^{2}\right\} \\
& \cdot \sin \left[2 \pi f_{m}\left(t-\frac{4}{f_{e}}\right)\right] \delta\left(x-x_{s}\right) \widehat{\mathbf{y}},
\end{aligned}
$$

where $f_{e}=20 \mathrm{GHz}$ and $f_{m}=5 \mathrm{GHz}$ are selected in order to obtain a spectral bandwidth ranging from $f_{\text {min }}=2 \mathrm{GHz}$ to $f_{\text {max }}=20 \mathrm{GHz}$.
The time and spatial extension of the computational domain are $t_{\text {tot }}=1 \mathrm{~ns}$ and $x_{\text {tot }}=10 \mathrm{~cm}$, respectively, the thickness of the biological media is $d=8 \mathrm{~cm}$, and the time and spatial steps are $\Delta t=0.18$ ps and $\Delta x=0.1 \mathrm{~mm}$, respectively. Moreover, the computational domain is closed from both sides by dedicated uniaxial perfectly matched layers [21].

The relative electric permittivity considered in the simulations is

$$
\begin{aligned}
\varepsilon_{r}(x, \omega)= & \varepsilon_{\infty}(x) \\
& +\frac{\varepsilon_{s}(x)-\varepsilon_{\infty}(x)}{1+0.43\left(\mathrm{j} \omega \tau_{0}\right)^{0.45}+0.13\left(\mathrm{j} \omega \tau_{0}\right)^{0.75}},
\end{aligned}
$$

where $\tau_{0}=15.9 \mathrm{ps}, \varepsilon_{\infty}(x)$ and $\varepsilon_{s}(x)$ are evaluated according (18)-(19), and $\varepsilon_{s, 1}=400, \varepsilon_{s, m}=20, \varepsilon_{\infty, 1}=10$, and $\varepsilon_{\infty, m}=$ 2. Therefore, a linear and exponential filling factor functions $f(x)$ are considered as follows:

$$
\begin{aligned}
& f(x)=f(0)+\frac{f(d)-f(0)}{d} x, \\
& f(x)=f(0) \exp \left\{-\frac{3 x}{d}\right\}
\end{aligned}
$$

where $f(0)$ is the initial value at the points $x=0$ and $f(d)=$ 0 .

In order to test the effectiveness of the presented fractional power series approach, a benchmarking against the multiterm Debye approximation and Padé approximation has been carried out. In particular, the relative permittivity 


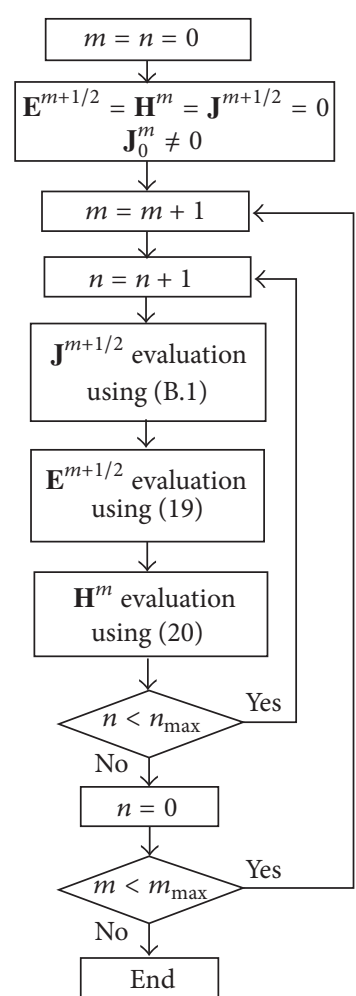

FIGURE 1: A flowchart of the proposed FDTD algorithm.

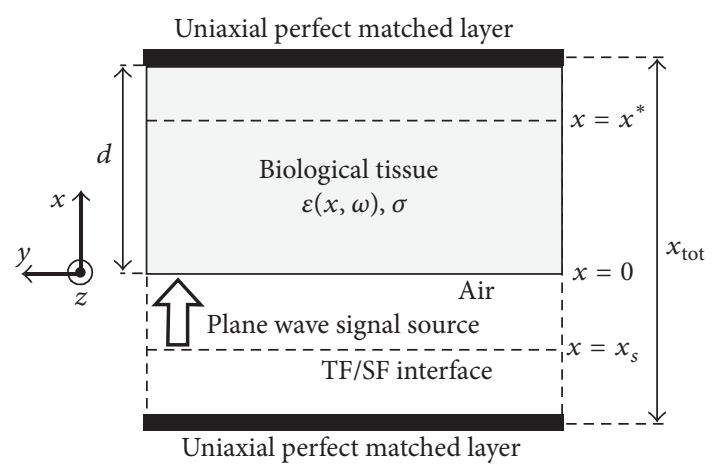

Figure 2: Slab model employed in the simulations.

function (21) with $\varepsilon_{\infty}(x)=10$ and $\varepsilon_{s}(x)=400$ has been approximated as follows:

$$
\begin{aligned}
& \varepsilon_{r}^{(D)}(\omega)=\varepsilon_{\infty}+\sum_{u=1}^{U} \frac{\Delta \varepsilon_{u}}{1+\mathrm{j} \omega \tau_{u}}, \quad \text { multiterm Debye, } \\
& \varepsilon_{r}^{(P)}(\omega)=\varepsilon_{\infty}+\frac{\sum_{u=0}^{U} \mathscr{A}_{u}\left(\mathrm{j} \omega \tau_{0}\right)^{u}}{\sum_{v=0}^{V} \mathscr{A}_{v}\left(\mathrm{j} \omega \tau_{0}\right)^{v}}, \quad \text { Padé. }
\end{aligned}
$$

To quantify the accuracy of the different approaches, the rootmean-square deviation on the complex electric permittivity
TABLE 1: Root-mean-square error obtained using the Debye and Padé-type approximations.

\begin{tabular}{lccc}
\hline Debye approximation & {$[U]$} & Padé approximation & {$[U / V]$} \\
\hline $8.97 \times 10^{-2}$ & {$[1]$} & $2.04 \times 10^{-1}$ & {$[1 / 1]$} \\
$1.25 \times 10^{-2}$ & {$[2]$} & $4.2 \times 10^{-2}$ & {$[2 / 2]$} \\
$3.9 \times 10^{-3}$ & {$[3]$} & $1.8 \times 10^{-2}$ & {$[3 / 3]$} \\
$1.1 \times 10^{-3}$ & {$[4]$} & $1.53 \times 10^{-2}$ & {$[4 / 4]$} \\
$4.6 \times 10^{-4}$ & {$[5]$} & $8 \times 10^{-3}$ & {$[5 / 5]$} \\
\hline
\end{tabular}

has been evaluated as

$$
\hat{f}_{\text {err }}=\sqrt{\frac{\int_{\omega_{\min }}^{\omega_{\max }}\left|\varepsilon_{r}(\omega)-\varepsilon_{r}^{(q)}(\omega)\right|^{2} \mathrm{~d} \omega}{\int_{\omega_{\min }}^{\omega_{\max }}\left|\varepsilon_{r}(\omega)\right|^{2} \mathrm{~d} \omega}},
$$

where the index $q=D, P$ identifies the specific type of approximation. As it can be noticed in Table 1, higher order of both Debye and Padé approximations results in a smaller fitting errors but at the same time the computational model becomes more complex. On the other hand, fractional power expansions lead to all-pole approximation of permittivity function. In this way, benefits in terms of memory usage and FDTD numerical implementation are achieved.

In order to validate the proposed approach, the numerical results given by the FDTD scheme are compared with those derived using a fully analytical frequency domain technique [34]. To this aim, the total field/scattered field (TF/SF) formulation, detailed in [24], has been implemented. TF/SF boundary separates the computational domain into a total field region, containing both the incident and scattered fields, and a scattered field region, containing only the scattered field. The TF/SF boundary can, in principle, be used to introduce any type of incident field into the FDTD grid, but, in practice, it is used almost exclusively to introduce plane wave excitation.

The simulations have been performed by considering a biological media described by (18), (21), and (23), where $f(0)=0.3$ and $N_{1}=0$ and $N_{2}=N_{3}=0.5$ (needles). In particular, the reflectance, $|R|^{2}$, and transmittance, $|T|^{2}$, have been calculated, where the reflection, $R$, and transmission, $T$, coefficients have been evaluated as follows:

$$
\begin{array}{r}
R(\omega)=\frac{E_{r}\left(\omega, x_{s}\right)}{E\left(\omega, x_{s}\right)}, \\
T\left(\omega, x^{*}\right)=\frac{E\left(\omega, x^{*}\right)}{E\left(\omega, x_{s}\right)},
\end{array}
$$

where $E_{r}\left(\omega, x_{s}\right)$ is the electric field reflected at the airbiological tissue interface, $E\left(\omega, x^{*}\right)$ is the electric field excited at the location $x^{*}=1 \mathrm{~cm}$, with $x^{*}$ being inside the biological tissue, and $E\left(\omega, x_{s}\right)$ is the electric field excited at the source location $x=x_{s}$.

Figure 3(a) shows the space-time distribution of the electric field resulting from the developed FDTD-based procedure. It worthwhile to note the transmission process 


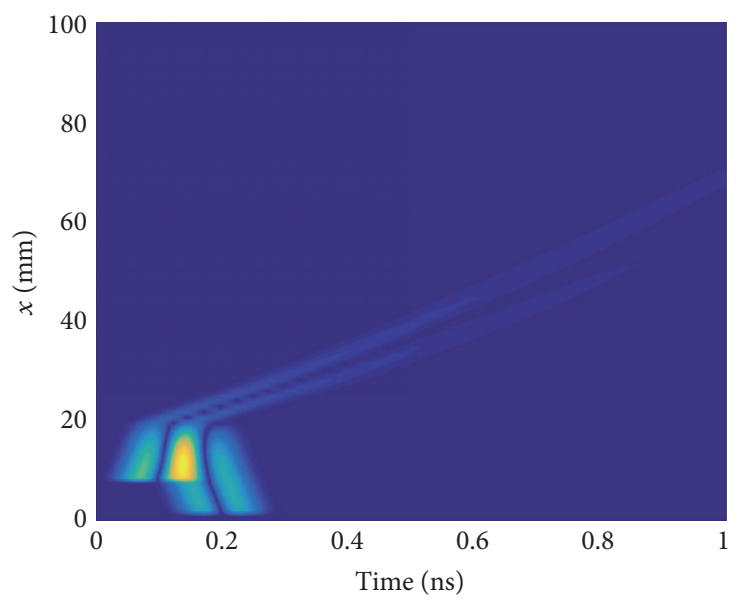

(a)

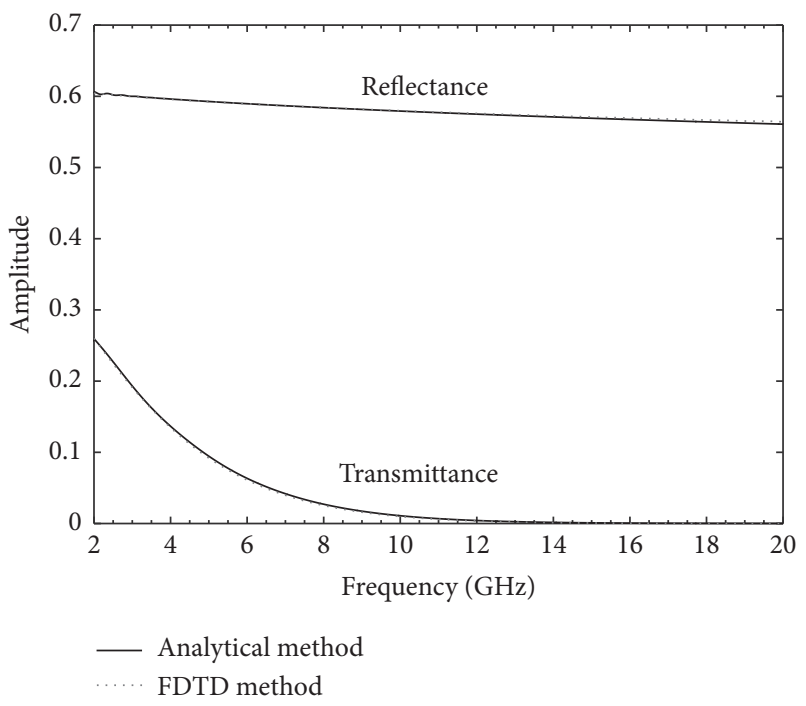

(b)

FIgURE 3: Validation test: (a) space-time electric field distribution and (b) reflectance and transmittance spectra.

as well as the reflection phenomenon occurring at the airbiological tissue interface. Moreover, the pulse spreading due to the propagation inside the dispersive biological media as well as the spatial variation of the group velocity due to the spatial dependence of the biological tissue permittivity is evident. Figure 3(b) illustrates the reflectance and transmittance spectra as evaluated by using the proposed FDTD procedure and the analytical technique. The excellent agreement between them fully validates the developed FDTD formulation.

To further investigate the accuracy and flexibility of the proposed FDTD scheme, the pulse propagation in complex media involving several spatial-dependent permittivity profiles has been studied. The numerical simulations have been performed by changing the filling factor function, the depolarization factor, and the mixing model. In particular, besides the function profiles (22)-(23) and the MaxwellGarnet and Bruggeman model, the depolarization factor sets $N_{1}=N_{2}=N_{3}=1 / 3$ (sphere), $N_{1}=0, N_{2}=N_{3}=1 / 2$ (needle), and $N_{1}=1, N_{2}=N_{3}=0$ (disc) are taken into account. In addition to the transmittance and reflectance, the penetration depth spectrum is calculated, too. This parameter is used to denote the depth at which the power density has decreased to $37 \%$ of its initial value at the surface.

The first test case regards the pulse propagation inside a dispersive medium described by the Maxwell-Garnet model, the linear filling factor function, and the depolarization factor $N_{1}=N_{2}=N_{3}=1 / 3$. The calculated numerical results are illustrated in Figure 4. By an inspection of Figure 4(a) it can be observed that the reflectance increases by increasing the initial value $f(0)$. In fact, considering that both $\varepsilon_{s, 1}$ and $\varepsilon_{\infty, 1}$ are higher than $\varepsilon_{s, m}$ and $\varepsilon_{\infty, m}, f(0)$ rising generates higher permittivity values resulting in an improvement of the permittivity variation at the air-biological tissue interface. On the other hand, lower values of $f(0)$ enable a better penetration of the electromagnetic field inside the biological media and as shown in Figure 4(b), the penetration depth increases. Moreover, the different relative variations of the transmittance and penetration depth at low and high frequency can be explained by considering that $\left(\varepsilon_{s, 1}-\varepsilon_{s, m}\right) \gg$ $\left(\varepsilon_{\infty, 1}-\varepsilon_{\infty, m}\right)$.

The second test case regards the pulse propagation inside a dispersive medium described by both the Maxwell-Garnet and Bruggeman models. The linear filling factor function, with $f(0)=0.6$, and the depolarization factor $N_{1}=$ $N_{2}=N_{3}=1 / 3$ are considered to perform the simulations. The obtained reflectance and transmittance as well as the penetration depth are illustrated in Figures 5(a) and 5(b), respectively. Differences in the propagation characteristics can be noticed especially at low frequency. Such result highlights a critical point regarding the choice of the suitable model describing the inhomogeneous permittivity function. In fact, for denser composites Bruggeman's formula is better suited than the Maxwell-Garnett one.

The third test case pertains the pulse propagation inside a dispersive medium described by both the linear and exponential filling factor functions having $f(0)=0.6$. The Maxwell-Garnet model and the depolarization factor $N_{1}=$ $N_{2}=N_{3}=1 / 3$ are considered, too. Figures $6(\mathrm{a})$ and 6(b) depict the transmittance/reflectance and penetration depth spectra, respectively. Since $f(0)$ is the same in linear and exponential functions, negligible reflectance variations occur. More significant transmittance and penetration depth changes can be observed since their trend mainly depends on the filling factor function.

The forth test case regards the pulse propagation inside a dispersive medium modeled by the depolarization factor sets $\mathcal{N}_{1}=\left\{N_{1}=N_{2}=N_{3}=1 / 3\right\}$ (sphere), $\mathcal{N}_{2}=\left\{N_{1}=0\right.$, $\left.N_{2}=N_{3}=1 / 2\right\}$ (needle), and $\mathcal{N}_{3}=\left\{N_{1}=1, N_{2}=\right.$ $\left.N_{3}=0\right\}$ (disc). Linear filling factor function having $f(0)=$ 0.6 and the Maxwell-Garnet model are considered, too. The calculated reflectance/transmittance and penetration depth 


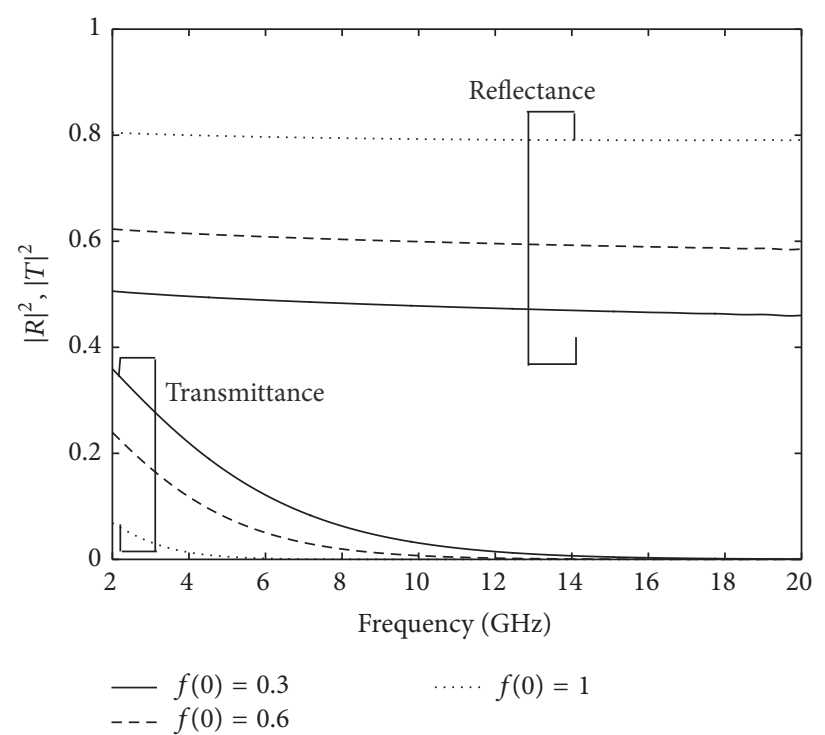

(a)

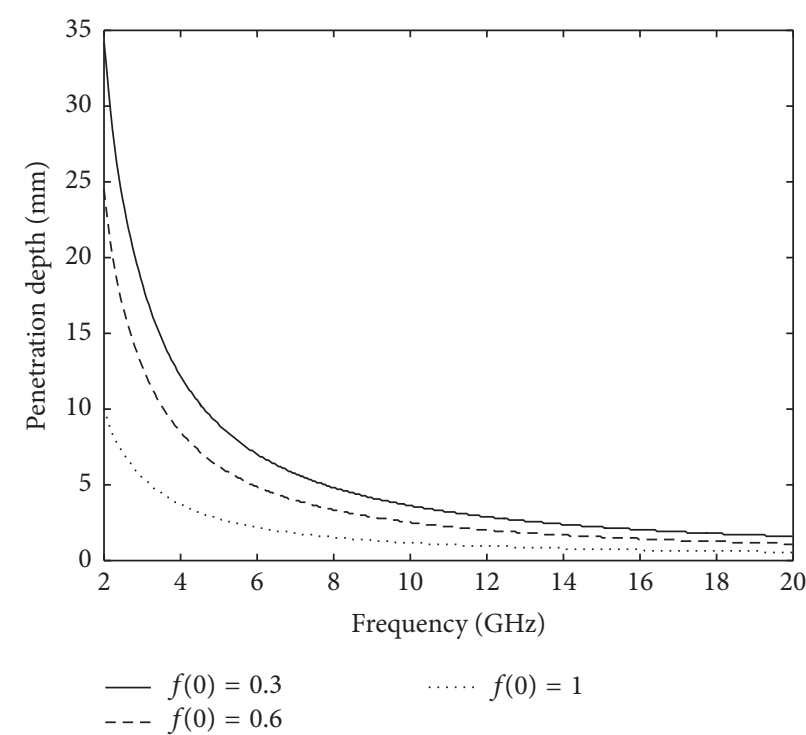

(b)

Figure 4: Pulse propagation characteristics inside dispersive media modeled by Maxwell-Garnet formula and described by linear filling factor function and depolarization factor $N_{1}=N_{2}=N_{3}=1 / 3$ : (a) reflectance and transmittance and (b) penetration depth spectra for different values of the initial value $f(0)$.

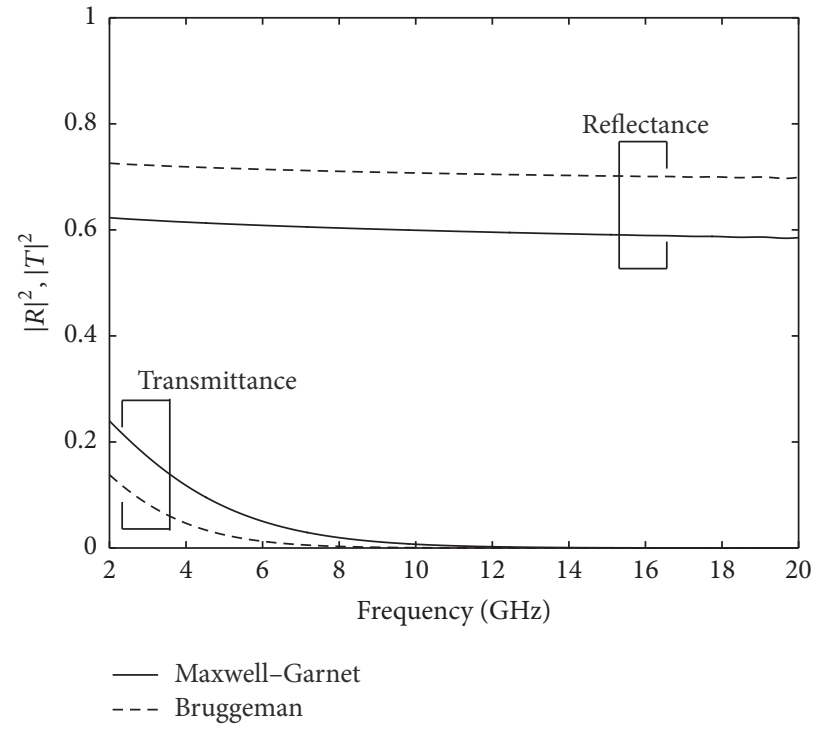

(a)

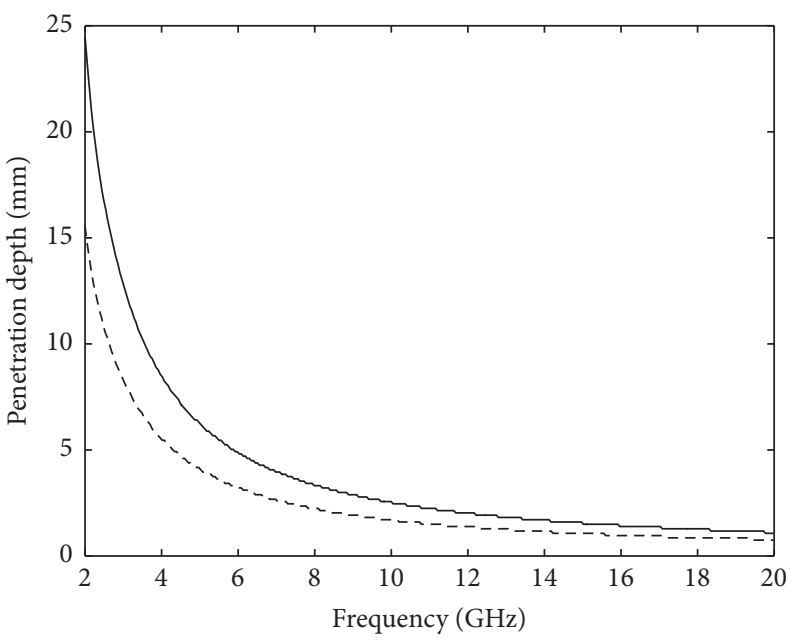

- Maxwell-Garnet

- - - Bruggeman

FIGURE 5: Pulse propagation characteristics inside dispersive media modeled by Maxwell-Garnet and Bruggeman models: (a) reflectance and transmittance and (b) penetration depth spectra. Depolarization factor $N_{1}=N_{2}=N_{3}=1 / 3$.

spectra are reported in Figures 7(a) and 7(b), respectively. By an inspection of these figures it is clear that the depolarization factors strongly affect the propagation properties of the dispersive media since the geometry and the surface area change the material response. In fact, the spherical shape creates the smallest dipole moment and when the spherical symmetry is strongly broken, as it happen for extremely squeezed ellipsoids (discs, needles), a quite strong deviation from the polarizability of the spherical shape occurs.
The final test case is the more general one since it pertains the electromagnetic analysis of a dispersive media characterized by a piecewise linear filling factor function:

$$
\begin{aligned}
& f(x) \\
& = \begin{cases}\frac{f\left(d_{1}\right)-f(0)}{d_{1}} x+f(0), & 0 \leq x \leq d_{1}, \\
\frac{f(d)-f\left(d_{1}\right)}{d-d_{1}} x+\frac{d f\left(d_{1}\right)-d_{1} f(d)}{d-d_{1}}, & d_{1}<x \leq d,\end{cases}
\end{aligned}
$$




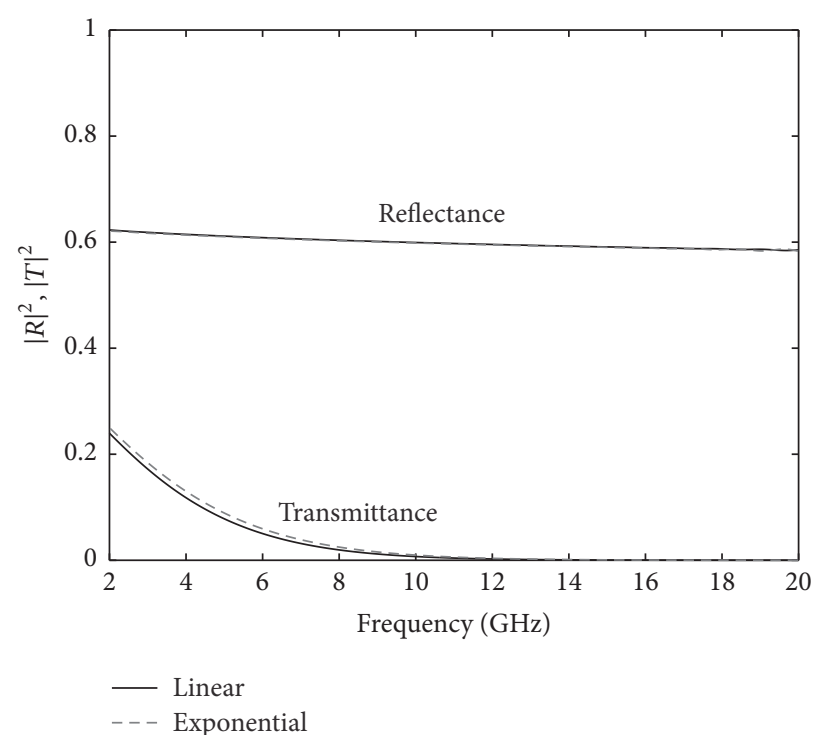

(a)

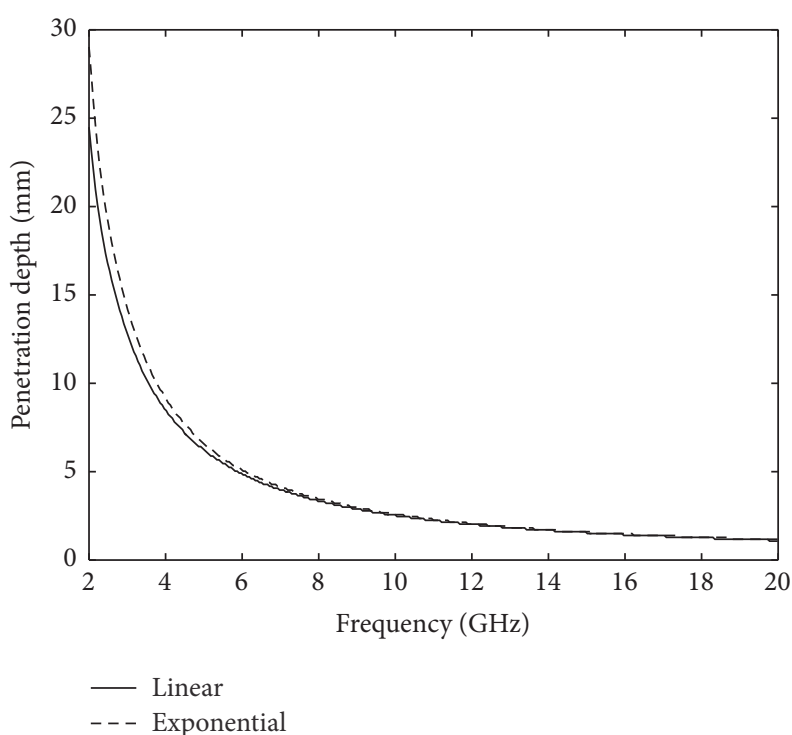

(b)

FIGURE 6: Pulse propagation characteristics inside dispersive media modeled by linear and exponential filling factor functions: (a) reflectance and transmittance and (b) penetration depth spectra. Depolarization factor $N_{1}=N_{2}=N_{3}=1 / 3$, Maxwell-Garnet model.

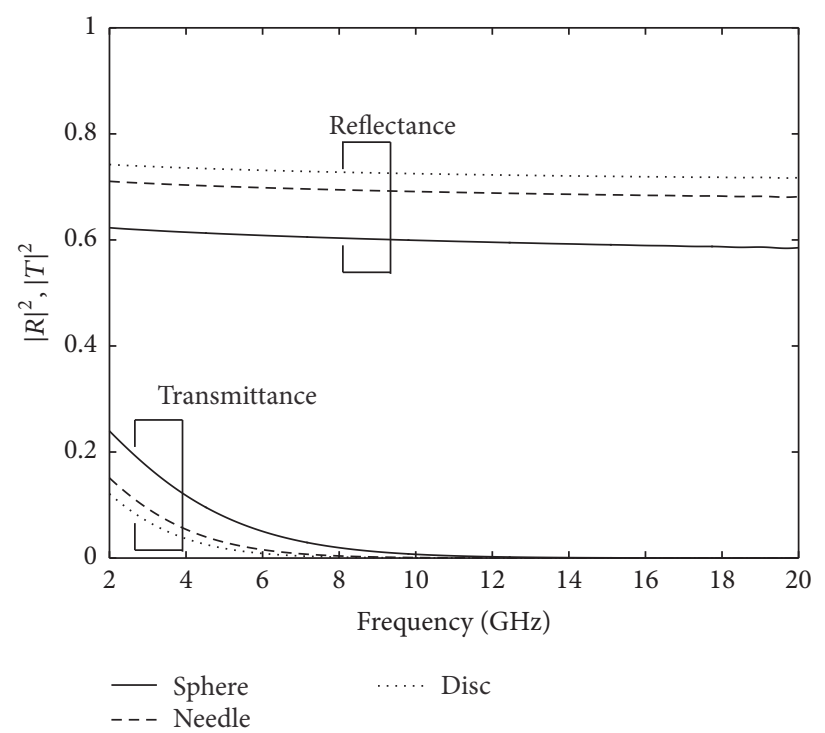

(a)

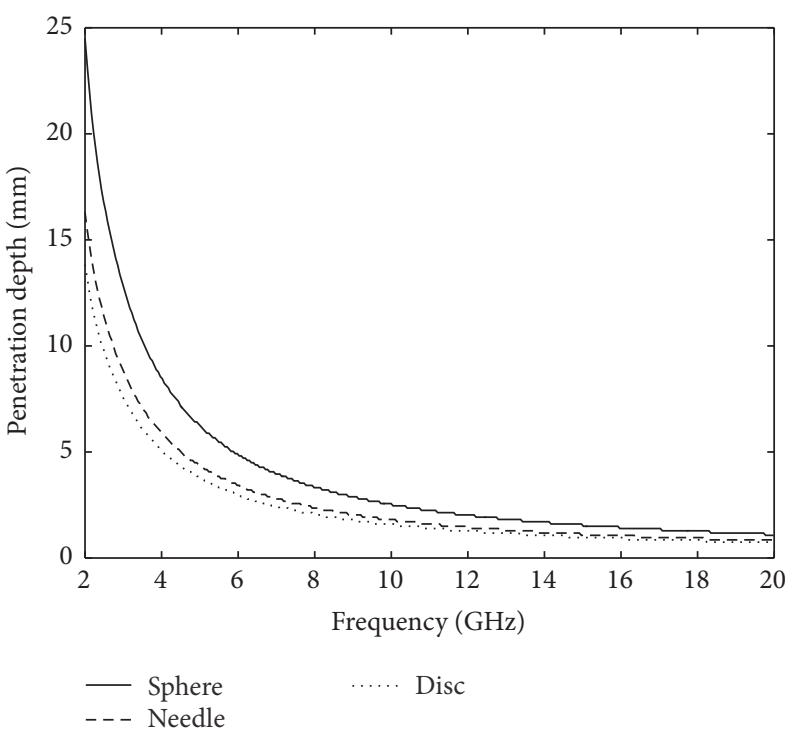

(b)

FIGURE 7: Pulse propagation characteristics inside dispersive media modeled by different depolarization factors: (a) reflectance and transmittance and (b) penetration depth spectra. Linear filling factor function having $f(0)=0.6$, Maxwell-Garnet model.

where $d_{1}=17 \mathrm{~cm}$ and $d=34 \mathrm{~cm}$. In particular, the coefficient sets $\mathscr{F}_{1}=\left\{f(0)=0, f\left(d_{1}\right)=0.6, f(d)=0.7\right\}$ and $\mathscr{F}_{2}=\left\{f(0)=1, f\left(d_{1}\right)=0.4, f(d)=0.3\right\}$ are simulated. Moreover, the Maxwell-Garnet model, the permittivity values $\varepsilon_{s, 1}=\varepsilon_{\infty, 1}=1, \varepsilon_{s, m}=20$, and $\varepsilon_{\infty, m}=2$, and the depolarization factor $N_{1}=N_{2}=N_{3}=1 / 3$ are also considered. Figure 8 shows the space-time distribution of the electric field as calculated by means of the developed FDTD technique. Figure 8(a) pertains the coefficient set $\mathscr{F}_{1}$ which generates a growing piecewise function composed by two linear functions having different slopes. In this case, the resulting permittivity decreases from its maximum value at $x=0$ as the $x$ coordinate increases. Moreover, a quite high permittivity gap at the interface $x=0$ occurs. As a result, the incident plane wave is strongly reflected by the airbiological tissue interface and the speed of the transmitted wave increases as its penetration inside the biological media increases. Figure $8(\mathrm{~b})$ pertains the coefficient set $\mathscr{F}_{2}$ which 


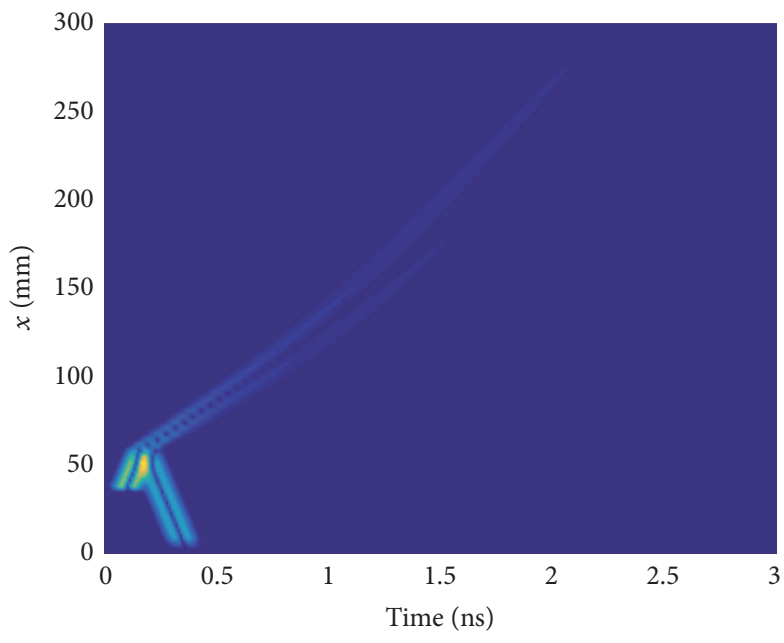

(a)

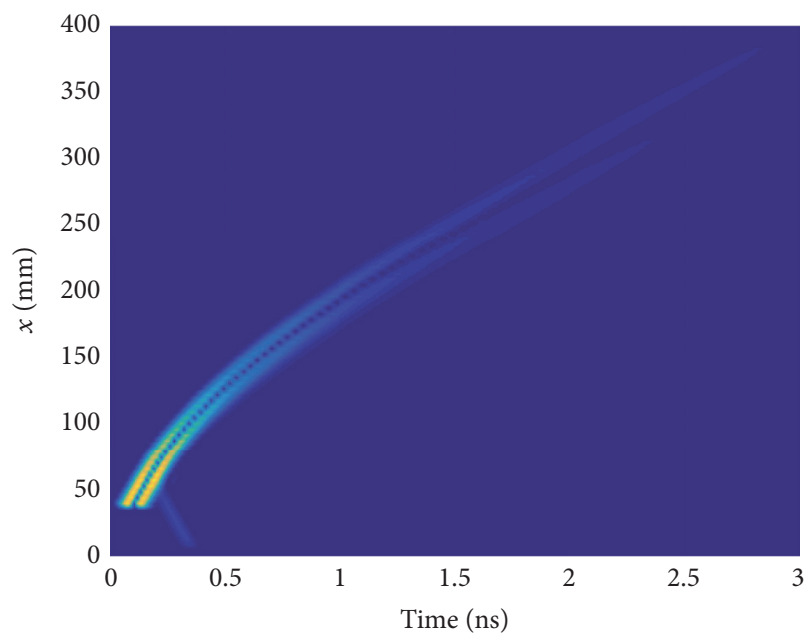

(b)

Figure 8: Space-time electric field distribution inside dispersive media modeled by piecewise linear filling factor function: (a) $f(0)=0$, $f\left(d_{1}\right)=0.6$, and $f(d)=0.7$ and (b) $f(0)=1, f\left(d_{1}\right)=0.4$, and $f(d)=0.3$.

generates a decreasing piecewise function. In this case, the permittivity of the biological medium at $x=0$ is well matched to the air permittivity and the incident plane wave is poorly reflected at the interface $x=0$. Moreover, because of the permittivity increasing as a function of the $x$ coordinate, the transmitted wave slowly penetrates inside the dispersive media.

\section{Conclusion}

An accurate FDTD model for simulating the pulse propagation in arbitrary dispersive media is proposed. Using the fractional derivative operator, the fractional part resulting from a general series expansion of the permittivity function of dispersive materials is directly incorporated into the FDTD scheme, avoiding the use of auxiliary differential equations. Moreover, dedicated uniaxial perfectly matched layer boundary conditions are derived and total field/scattered field formulation is adopted in combination with the basic timemarching scheme. As a result, the newly proposed algorithm gets excellent accuracy with minimum auxiliary variables and thus minimum storage spaces. Its application in modeling the electromagnetic pulse propagation in several test cases is presented and simulation results demonstrate the reliability and efficiency of the proposed method.

Such a technique can be considered as a general scheme for modeling various types of dispersions including Debye, Cole-Cole, Cole-Davidson, Havriliak-Negami, Drude-Lorentz, and universal dielectric response. The presented FDTD scheme can be considered as a general-purpose tool useful to address complex electromagnetic problems in the field of bioengineering. It could be successfully applied in the field of remotely powered implantable devices for optimizing the trade-off between the received power and tissue absorption. It could be also useful to evaluate in detail the temperature elevation in the biological tissues as well as to perform an accurate investigation of the spatial and temporal distribution of the transmembrane voltage and electroporation phenomenon in cells exposed to high voltage electric pulses. Finally, it could be extended to perform an extensive numerical analysis of pulsed electromagnetic fields propagation in colloids, emulsions, and random dielectric materials.

\section{Appendix}

\section{A. Fourier Transform of the Power-Law Function}

For a real number $\beta>-1$, the gamma function is defined as

$$
\Gamma(\beta+1)=\int_{0}^{\infty} t^{\beta} \mathrm{e}^{-t} \mathrm{~d} t
$$

The Fourier transform of the power-law function is

$$
\mathscr{F}\left\{t^{\beta}\right\}=\int_{0}^{\infty} t^{\beta} \mathrm{e}^{-\mathrm{j} \omega t} \mathrm{~d} t,
$$

where $t^{\beta}=0$ for $t<0$, and using the variable change $u=j \omega t$, it is straightforward to obtain

$$
\begin{aligned}
\mathscr{F}\left\{t^{\beta}\right\} & =(\mathrm{j} \omega)^{-\beta-1} \int_{0}^{\infty} u^{\beta} \mathrm{e}^{-u} \mathrm{~d} u \\
& =(\mathrm{j} \omega)^{-\beta-1} \Gamma(\beta+1) .
\end{aligned}
$$

The inverse Fourier transform of (A.3) gives

$$
\mathscr{F}^{-1}\left\{\mathscr{F}\left\{t^{\beta}\right\}\right\}=t^{\beta}=\Gamma(\beta+1) \int_{-\infty}^{\infty}(\mathrm{j} \omega)^{-\beta-1} \mathrm{e}^{\mathrm{j} \omega t} \mathrm{~d} \omega
$$

and comparing (A.4) with (8), (9) can be easily achieved. 


\section{B. Time-Marching Scheme}

Evaluating (10) at $t=m \Delta t$ yields

$$
\left.\mathbf{J}\right|^{m}+\left.\sum_{p=1}^{N} \int_{0}^{t} \zeta_{p}\left(t^{\prime}\right) \frac{\partial^{v_{p}} \mathbf{J}}{\partial t^{v_{p}}}\right|^{m} \mathrm{~d} t^{\prime}=\left.\varepsilon_{0} \Delta \varepsilon \frac{\partial \mathbf{E}}{\partial t}\right|^{m},
$$

where

$$
\begin{aligned}
\left.\frac{\partial^{v_{p}} \mathbf{J}}{\partial t^{v_{p}}}\right|^{m} \approx & \left.\frac{1}{(\Delta t)^{v_{p}}} \sum_{n=0}^{v_{p}}(-1)^{n}\left(\begin{array}{c}
v_{p} \\
n
\end{array}\right) \mathbf{J}\right|^{m-n+1 / 2} \\
\approx & \frac{1}{(\Delta t)^{v_{p}}} \mathbf{J}^{m+1 / 2} \\
& +\left.\frac{1}{(\Delta t)^{v_{p}}} \sum_{n=1}^{v_{p}}(-1)^{n}\left(\begin{array}{c}
v_{p} \\
n
\end{array}\right) \mathbf{J}\right|^{m-n+1 / 2} .
\end{aligned}
$$

But

$$
\begin{aligned}
& \left.\int_{0}^{t} \zeta_{p}\left(t^{\prime}\right) \frac{\partial^{v_{p}} \mathbf{J}}{\partial t^{v_{p}}}\right|^{m} \mathrm{~d} t^{\prime} \\
& =\left.\sum_{k=0}^{m-1} \frac{\partial^{v_{p}} \mathbf{J}}{\partial t^{v_{p}}}\right|^{m-k} \int_{k \Delta t}^{(k+1) \Delta t} \zeta_{p}\left(t^{\prime}\right) \mathrm{d} t^{\prime} \\
& =\left.\sum_{k=0}^{m-1} \eta_{p, k} \frac{\partial^{v_{p}} \mathbf{J}}{\partial t^{v_{p}}}\right|^{m-k},
\end{aligned}
$$

where

$$
\begin{aligned}
\eta_{p, k} & =\int_{k \Delta t}^{(k+1) \Delta t} \zeta_{p}\left(t^{\prime}\right) \mathrm{d} t^{\prime} \\
& =\frac{A_{p} \tau_{0}^{\alpha_{p}}(\Delta t)^{1-\xi_{p}}}{\left(1-\xi_{p}\right) \Gamma\left(1-\xi_{p}\right) \Delta t}\left[(k+1)^{1-\xi_{p}}-k^{1-\xi_{p}}\right] \\
& =\frac{A_{p}\left(\tau_{0} / \Delta t\right)^{\alpha_{p}}}{\Gamma\left(2-\xi_{p}\right)(\Delta t)^{1-v_{p}}}\left[(k+1)^{1-\xi_{p}}-k^{1-\xi_{p}}\right] .
\end{aligned}
$$

Rearranging (14) and using (15) and (B.1)-(B.4) give

$$
\begin{aligned}
& {\left.\left[\frac{1}{2}+\frac{\varepsilon_{\infty}}{\Delta \varepsilon}\left(R+\frac{1}{2}\right)\right] \mathbf{J}\right|^{m+1 / 2}=\left.(\nabla \times \mathbf{H})\right|^{m}-\left.\mathbf{J}_{0}\right|^{m}} \\
& -\left.\frac{1}{2}\left(1+\frac{\varepsilon_{\infty}}{\Delta \varepsilon}\right) \mathbf{J}\right|^{m-1 / 2} \\
& -\frac{\varepsilon_{\infty}}{\Delta \varepsilon_{\infty}}\left[\left.\sum_{p=1}^{N} \sum_{n=1}^{v_{p}} \frac{\eta_{p, 0}}{(\Delta t)^{v_{p}}}(-1)^{n}\left(\begin{array}{c}
v_{p} \\
n
\end{array}\right) \mathbf{J}\right|^{m-n+1 / 2}\right. \\
& +Q]+-\sigma\left(\frac{\left.\mathbf{E}\right|^{m-1 / 2}+\left.\mathbf{E}\right|^{m+1 / 2}}{2}\right),
\end{aligned}
$$

where

$$
\begin{aligned}
& Q=\left.\sum_{p=1}^{N} \sum_{k=1}^{m-1} \frac{\eta_{p, k}}{(\Delta t)^{v_{p}}} \frac{\partial^{v_{p}} \mathbf{J}}{\partial t^{v_{p}}}\right|^{m-k}, \\
& R=\sum_{p=1}^{N} \frac{\eta_{p, 0}}{(\Delta t)^{v_{p}}} .
\end{aligned}
$$

Rearranging (B.1) gives

$$
\begin{aligned}
& \left.\mathbf{E}\right|^{m+1 / 2}=\left.\mathbf{E}\right|^{m-1 / 2}+\left.\frac{\Delta t}{\varepsilon_{0} \Delta \varepsilon}\left(R+\frac{1}{2}\right) \mathbf{J}\right|^{m+1 / 2} \\
& +\left.\frac{\Delta t}{2 \varepsilon_{0} \Delta \varepsilon} \mathbf{J}\right|^{m-1 / 2} \\
& +\frac{\Delta t}{\varepsilon_{0} \Delta \varepsilon}\left[\left.\sum_{p=1}^{N} \sum_{n=1}^{v_{p}} \frac{\eta_{p, 0}}{(\Delta t)^{v_{p}}}(-1)^{n}\left(\begin{array}{c}
v_{p} \\
n
\end{array}\right) \mathbf{J}\right|^{m-n+1 / 2}\right. \\
& +Q] .
\end{aligned}
$$

\section{Competing Interests}

The authors declare that they have no competing interests.

\section{Acknowledgments}

This work has been partially supported within the "Aiuti a Sostegno dei Cluster Tecnologici Regionali” plan: LPIJ9P2 PERFOrm TECH-Puglia Emerging FOOD Technology.

\section{References}

[1] D. Kissinger and J.-C. Chiao, "Medical applications of radiofrequency and microwaves-sensing, monitoring, and diagnostics," IEEE Microwave Magazine, vol. 16, no. 4, pp. 34-38, 2015.

[2] A. V. Vorst, A. Rosen, and Y. Koysuka, RF/Microwave Interaction with Biological Tissues, John Wiley \& Sons, New Jersey, NJ, USA, 2006.

[3] J. Chiao and D. Kissinge, "Medical applications of rf and microwaves-therapy and safety," IEEE Micro Magazine, vol. 16, pp. 12-13, 2015.

[4] K. Grenier, D. Dubuc, T. Chen et al., "Recent advances in microwave-based dielectric spectroscopy at the cellular level for cancer investigations," IEEE Transactions on Microwave Theory and Techniques, vol. 61, no. 5, pp. 2023-2030, 2013.

[5] M. Lazebnik, C. Zhu, G. M. Palmer et al., "Electromagnetic spectroscopy of normal breast tissue specimens obtained from reduction surgeries: comparison of optical and microwave properties," IEEE Transactions on Biomedical Engineering, vol. 55, no. 10, pp. 2444-2451, 2008.

[6] J. M. L. P. Caldeira, J. J. P. C. Rodrigues, and P. Lorenz, "Toward ubiquitous mobility solutions for body sensor networks on healthcare," IEEE Communications Magazine, vol. 50, no. 5, pp. 108-115, 2012.

[7] S. Movassaghi, M. Abolhasan, J. Lipman, D. Smith, and A. Jamalipour, "Wireless body area networks: a survey", IEEE Communications Surveys and Tutorials, vol. 16, no. 3, pp. 16581686, 2014.

[8] T. Nakano, M. J. Moore, F. Wei, A. V. Vasilakos, and J. Shuai, "Molecular communication and networking: opportunities and challenges," IEEE Transactions on Nanobioscience, vol. 11, no. 2, pp. 135-148, 2012.

[9] S.-C. Huang, J.-W. Kang, H.-W. Tsai, Y.-S. Shan, X.-Z. Lin, and G.-B. Lee, "Molecular communication and networking: opportunities and challenges," IEEE Transactions on Biomedical Engineering, vol. 61, no. 2, pp. 598-605, 2014. 
[10] M. Breton and L. M. Mir, "Microsecond and nanosecond electric pulses in cancer treatments," Bioelectromagnetics, vol. 33, no. 2, pp. 106-123, 2012.

[11] L. Debnath, "Recent applications of fractional calculus to science and engineering," International Journal of Mathematics and Mathematical Sciences, vol. 2003, no. 54, pp. 3413-3442, 2003.

[12] M. Dalir, "Applications of fractional calculus," Applied Mathematical Sciences, vol. 4, pp. 1021-1032, 2010.

[13] J. F. Douglas, "Some applications of fractional calculus to polymer science," Advances in Chemical Physics, vol. 102, pp. 121-192, 1997.

[14] V. V. Kulish and J. L. Lage, "Application of fractional calculus to fluid mechanics," Journal of Fluids Engineering, Transactions of the ASME, vol. 124, no. 3, pp. 803-806, 2002.

[15] R. Magin, Fractional Calculus in Bioengineering, Begell House, 2006.

[16] C. Polk and E. Postow, Biological Effects of Electromagnetic Fields, Crc Press, Boca Raton, Fla, USA, 1996.

[17] G. G. Raju, Dielectrics in Electric Fields, Marcel Dekker, New York, NY, USA, 2003.

[18] R. Pethig and D. B. Kell, "The passive electrical properties of biological systems: their significance in physiology, biophysics and biotechnology," Physics in Medicine and Biology, vol. 32, no. 8, pp. 933-970, 1987.

[19] W. D. Hurt, "Multiterm debye dispersion relations for permittivity of muscle," IEEE Transactions on Biomedical Engineering, vol. 32, no. 1, pp. 60-64, 1985.

[20] L. Mescia, P. Bia, and D. Caratelli, "Fractional-calculus-based FDTD method for solving pulse propagation problems," in Proceedings of the 17th IEEE International Conference on Electromagnetics in Advanced Applications (ICEAA '15), pp. 460-463, Torino, Italy, September 2015.

[21] L. Mescia, P. Bia, and D. Caratelli, "Fractional derivative based FDTD modeling of transient wave propagation in HavriliakNegami media," IEEE Transactions on Microwave Theory and Techniques, vol. 62, no. 9, pp. 1920-1929, 2014.

[22] P. Bia, D. Caratelli, L. Mescia, R. Cicchetti, G. Maione, and F. Prudenzano, "A novel FDTD formulation based on fractional derivatives for dispersive Havriliak-Negami media," Signal Processing, vol. 107, pp. 312-318, 2015.

[23] L. Mescia, P. Bia, and D. Caratelli, "Authors reply," IEEE Transactions on Microwave Theory and Techniques, vol. 63, no. 12, pp. 4191-4193, 2015.

[24] A. Taove and S. Hagness, Computational Electrodynamics: the Finite-Difference Time-Domain Method, Artech House, Norwood, Mass, USA, 2005.

[25] R. E. Collin, Field Theory of Guided Waves, Wiley-IEEE Press, New York, NY, USA, 1990.

[26] C. A. Balanis, Advanced Engineering Electromagnetics, John Wiley \& Sons, New York, NY, USA, 2012.

[27] W.-J. Chen, W. Shao, and B.-Z. Wang, "ADE-laguerre-FDTD method for wave propagation in general dispersive materials," IEEE Microwave and Wireless Components Letters, vol. 23, no. 5, pp. 228-230, 2013.

[28] M. F. Causley and P. G. Petropoulos, "On the time-domain response of Havriliak-Negami dielectrics," IEEE Transactions on Antennas and Propagation, vol. 61, no. 6, pp. 3182-3189, 2013.

[29] H. H. Abdullah, H. A. Elsadek, H. E. ElDeeb, and N. Bagherzadeh, "Fractional derivatives based scheme for FDTD modeling of nth-order Cole-Cole dispersive media," IEEE Antennas and Wireless Propagation Letters, vol. 11, pp. 281-284, 2012.
[30] W. H. Weedon and C. M. Rappaport, "A general method for FDTD modeling of wave propagation in arbitrary frequencydispersive media," IEEE Transactions on Antennas and Propagation, vol. 45, no. 3, pp. 401-410, 1997.

[31] D. Caratelli, L. Mescia, P. Bia, and O. V. Stukach, "Fractionalcalculus-based FDTD algorithm for ultrawideband electromagnetic characterization of arbitrary dispersive dielectric materials," IEEE Transactions on Antennas and Propagation, vol. 64, no. 8, pp. 3533-3544, 2016.

[32] P. Bia, D. Caratelli, L. Mescia, and J. Gielis, "Analysis and synthesis of supershaped dielectric lens antennas," IET Microwaves, Antennas and Propagation, vol. 9, no. 14, pp. 1497-1504, 2015.

[33] A. Shivola, Electromagnetic Mixing Formulas and Applications, vol. 47 of Electromagnetic Waves Series, IEE Publishing, London, UK, 1999.

[34] P. Yeh, Optical Waves in Layered Media, John Wiley \& Sons, New York, NY, USA, 1988. 


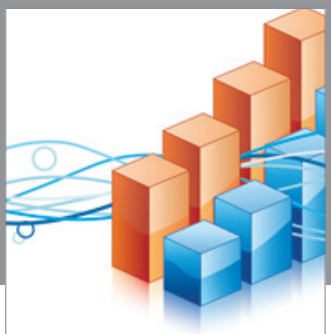

Advances in

Operations Research

vatem alat4

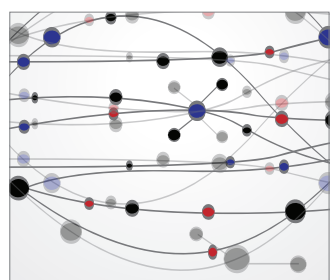

\section{The Scientific} World Journal
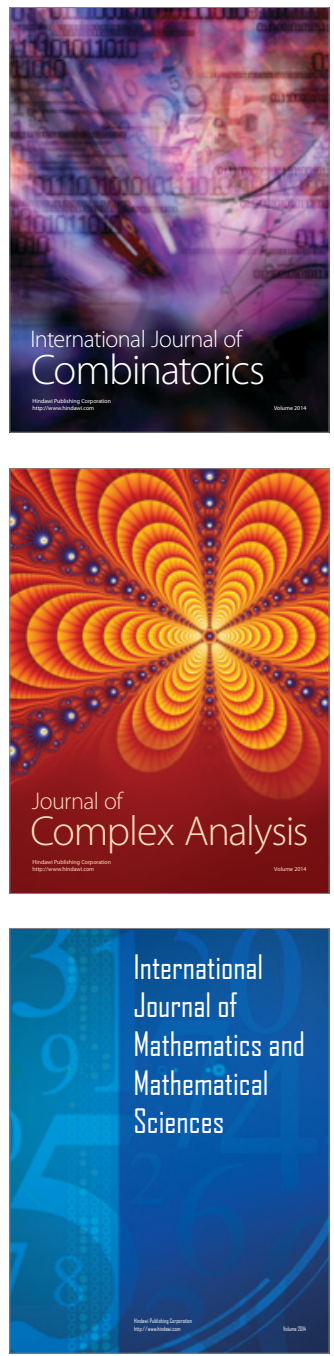
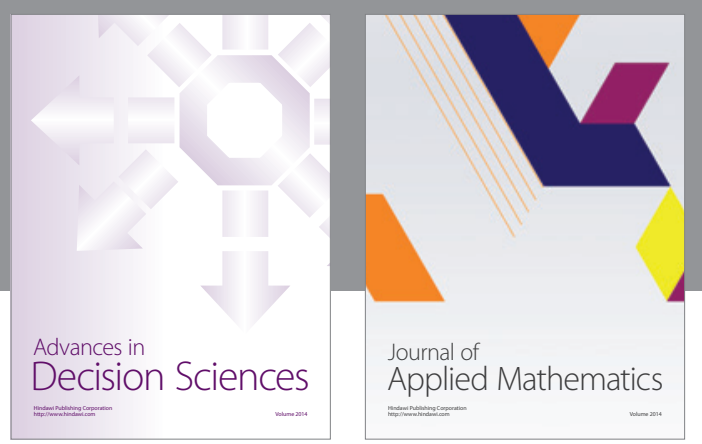

Algebra

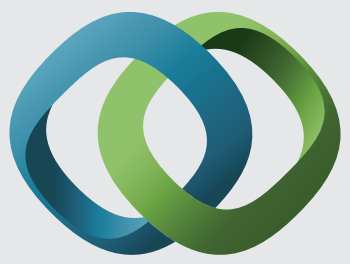

\section{Hindawi}

Submit your manuscripts at

http://www.hindawi.com
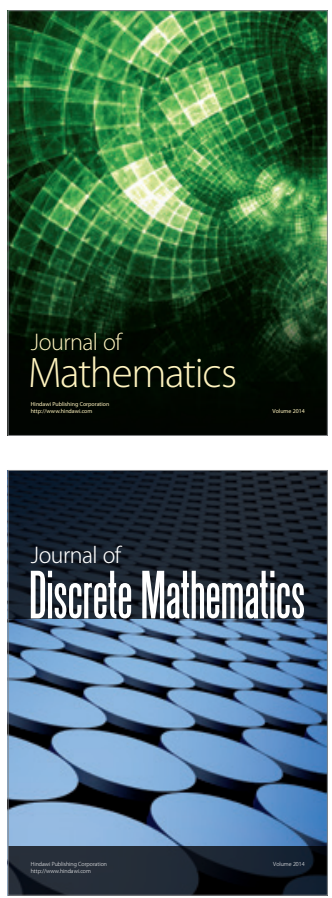

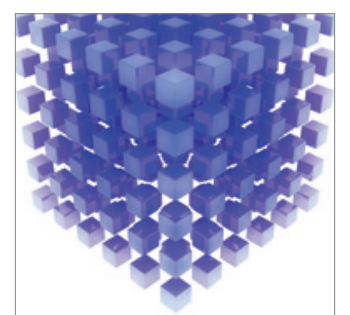

Mathematical Problems in Engineering
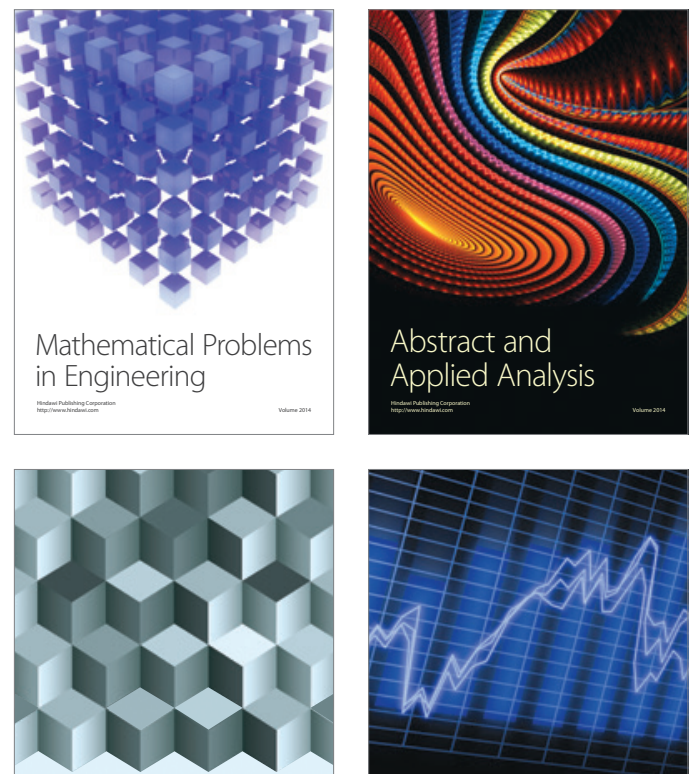

Journal of

Function Spaces

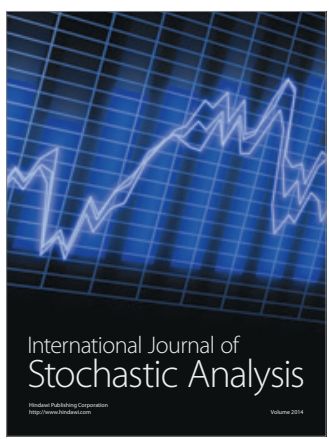

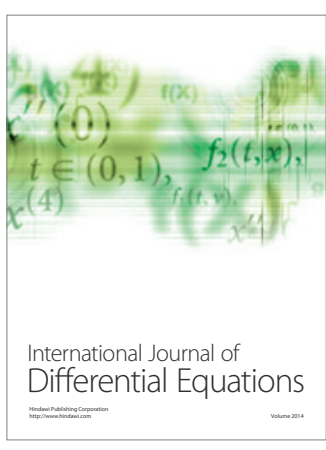
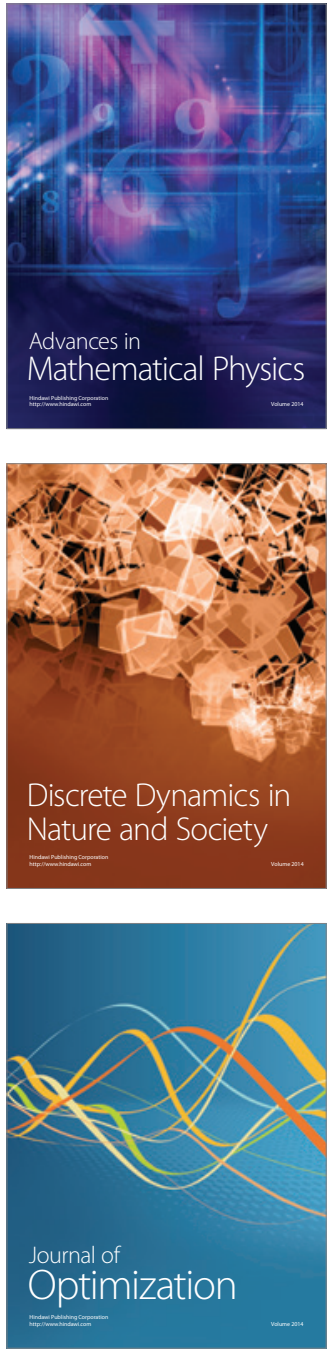